\title{
Small-footprint LiDAR Estimation of Structural Properties of Woody Plant Communities in Complex Terrain, North-eastern Australia
}

\author{
Ediriweera S. ${ }^{1 *}$, Danaher T. ${ }^{2}$, Pathirana $S .^{3}$ and Nichols D. ${ }^{3}$ \\ ${ }^{1}$ Uva Wellassa University, Badulla, Sri Lanka \\ ${ }^{2}$ New South Wales Department of Environment, Climate Change and Water, Australia \\ ${ }^{3}$ Southern Cross University, Australia \\ *sisira@uwu.ac.lk
}

\begin{abstract}
LiDAR remote sensing can be considered a key instrument for studies related to quantifying the vegetation structure. We utilised LiDAR metrics to estimate plot-scale structural parameters of subtropical rainforest and eucalyptus dominated open forest in topographically dissected landscape, in North-eastern Australia. This study is considered an extreme application of LiDAR technology for structurally complex subtropical forests in complex terrain. Thirty-one LiDAR metrics of vegetation functional parameters were examined. Multiple linear regression models were able to explain $62 \%$ of the variability associated with basal area, $66 \%$ for mean dbh, $61 \%$ for dominant height and $60 \%$ for foliage projective cover in subtropical rainforest. In contrast, mean height (adjusted $\mathrm{R}^{2}=0.90$ ) and dominant height (adjusted $\mathrm{R}^{2}=0.81$ ) were predicted with highest accuracy in the eucalyptus dominated open canopy forest. Nevertheless, the magnitude of error for predicting structural parameters of vegetation was much higher in subtropical rainforest than those documented in the literature. Our findings reinforced that obtaining accurate LiDAR estimates of vegetation structure is a function of the complexity of horizontal and vertical structural diversity of vegetation.
\end{abstract}

Keywords: LiDAR, Vegetation structure, Subtropical rainforest, Hilly terrain 